\title{
Retraction
}

\section{Author-Initiated Retraction: Anderson et al, Induced Alpha Rhythms Track the Content and Quality of Visual Working Memory Representations with High Temporal Precision}

At the request of the authors, The Journal of Neuroscience is retracting "Induced Alpha Rhythms Track the Content and Quality of Visual Working Memory Representations with High Temporal Precision” by David E. Anderson, John T. Serences, Edward K. Vogel, and Edward Awh, which appeared on pages 7587-7599 of the May 28, 2014 issue.

We regret that there was an error in the analytic code used to compute oscillatory power in our article. Specifically, there was a matrix transposition error in the code (see abs(hilbert(eegfilt(data,Fs,f1,f2))). $\hat{2}$ on page 7588 , right column, end of second full paragraph). The data matrix was oriented correctly for the call to eegfilt, but the output of the call to eegfilt was not correctly transposed in the standard Matlab format before passing into the built-in Matlab 'hilbert' function, as the EEGLAB function 'eegfilt' and the built-in Matlab function 'hilbert' require the data matrix to have different dimensions in order to operate correctly across time. Fortunately, this error had a relatively modest impact on the overall pattern of data, because even though the imaginary component of the complex modulus of the hilbert transformed data was incorrect, the real component was not affected and averaging across trials largely washed out the perturbations in our estimates of instantaneous power. Thus, because our analysis was focused on the spatial distribution of power, the broad empirical patterns that we reported remain intact even after the matrix transposition was corrected. The spatial distribution of alpha power across electrodes still covaries with the orientation of the remembered stimulus, and thus still enables time-resolved tracking of the contents of visual working memory (i.e., Fig. $2 A, C$ ), and this neural activity is sensitive to whether or not the observer is voluntarily maintaining the memorandum in working memory. Nevertheless, the corrected analysis no longer shows a robust correlation between behavioral mnemonic precision and the tuning properties of the neural activity as reported in the original article. Thus, we chose to retract the paper because it was evaluated by reviewers in the context of some findings that we no longer believe to be robust.

DOI: 10.1523/JNEUROSCI.0074-15.2015 